Ann. Biol. anim. Bioch. Biophys., 1979, 19 (2 B), 487-488.

\title{
A. Recherches anatomopathologiques chez le rat ingérant différentes doses d'huile d'arachide ou d'huile de colza à faible teneur en acide érucique (Huile de colza Primor). 3. Etude anatomopathologique des organes
}

par X. FOUILLET ( $\left.{ }^{1}\right)$

Cenire de Recherches et d'Elevoge des Oncins (I.F.R.E.B.)

69210 Saint Germain sur l'Arbresle.

Summary. A. Anatomo-pathological research on rats ingesting different doses of peanut oil or of low-erucic acid rapeseed oil (Primor rapeseed oil). 3. Anatomo-pathology of the organs.

Rats were fed a diet containing 5 p. 100 peanut oil or Primor rapeseed oil for 1 year. At the end of the experiment, there were no histological differences between the groups for the organs examined (lungs, liver, spleen, kidneys).

Le poumon, le foie, la rate et le rein des rats ayant reçu pendant 1 an 5 p. 100 d'huile d'arachide ou d'huile de colza Primor ont été examinés en vue d'une étude anatomopathologique.

Les conditions expérimentales et les méthodes ont été décrites précédemment.

\section{Résultats.}

Les données de l'examen histologique sont consignées dans le tableau suivant.

Huile d'arachide

\begin{tabular}{|c|c|c|c|c|c|c|c|c|c|c|c|}
\hline Animal No .. & 1 & 7 & 9 & 14 & 15 & 21 & 23 & 26 & 27 & 34 & 39 \\
\hline Poumon .... & 1 & 1 & 1 & 1 & 5 & 1 & 1 & 5 & 1 & 7 & 1 \\
\hline Foie $\ldots \ldots \ldots$ & 1 & 1 & 1 & 9 & 8 & 8 & 9 & 1 & 1 & 1 & 1 \\
\hline Rate ...... & 4 & 4 & 4 & 4 & 4 & 4 & 4 & 4 & 4 & 4 & 4 \\
\hline Reins & $1 a$ & $1 a$ & $1 b$ & $1 a$ & $1 b-3$ & 10 & $1 a$ & $1 c$ & $1 b$ & $1 c$ & $1 b$ \\
\hline
\end{tabular}

(1) Adresse actuelle : Centre de Recherche Battelle, 1227 Carouge-Genève, Suisse. 
Huile de colza Primor

\begin{tabular}{lccccccccccc}
\hline Animal No .. & 42 & 48 & 51 & 62 & 54 & 56 & 66 & 68 & 70 & 75 & 77 \\
\hline Poumon $\ldots \ldots$ & 7 & $/$ & $5-6$ & $/$ & $/$ & $/$ & 7 & 7 & 7 & $/$ & $/$ \\
\hline Foie......... & $/$ & $/$ & $/$ & $/$ & $/$ & $/$ & $/$ & $/$ & $/$ & $/$ & $/$ \\
\hline Rate $\ldots . . .$. & $4 a$ & 4 & 4 & 4 & $4 a$ & 4 & 4 & 4 & 4 & 4 & 4 \\
\hline Reins ....... & $1 b$ & $1 a$ & $1 b$ & $1 b$ & $1 b$ & $1 b$ & $1 c$ & $1 b$ & $1 c$ & $1 a-2$ & $1 d$ \\
\hline
\end{tabular}

Chaque chiffre, éventuellement suivi d'une lettre minuscule, renvoie à un type de lésion pour un organe donné.

I : Aspect histologique normal.

1 : Lésions rénales au niveau de la corticale. Banales chez le rał de laboratoire, elles sont décrites dans la littérature comme une néphrite interstitielle chronique. De nombreuses altérations des cellules pariétales des tubes contournés sont notées, accompagnées de cylindres hyalins dans les tubules, principalement à la jonction médullo-corticale. On note d'autre part un épaississement des basales tubulaires ef de nombreux infiltrats de cellules lymphoïdes dans l'interstitium. L'atteinte glomérulaire, avec épaississement de la capsule de Bowman, n'est remarquée que dans les cas les plus sévères (à partir de $1 c$ ). $1 a$ : lésions marquées $(2+) ; 1 b$ : lésions sévères $(3+) ; 1 c$ : lésions très sévères $(4+)$; $1 d$ : lésions extrêmement sévères $(5+)$.

2 : Dilałation de la cavité calicielle.

3 : Edème interstitiel à la pointe de la papille rénale.

4 : Stase sanguine marquée dans les sinus veineux de la pulpe rouge. Assez nombreux foyers d'hématopoièse extramédullaire au niveau des cordons de Billroth. $4 a$ : de plus, une intense rétention d'hémosidérine dans les macrophages.

5 : Plages de densification des parois alvéolaires.

6 : Petite zone de calcification de l'intima d'une artère pulmonaire.

7 : Plages d'alvéolite hémorragique.

8 : Par endroits, en région centrolobulaire surtout, plages d'hépatocytes macrovacuolisés (lipides probables).

9 : Rares hépatocyłes macrovacuolisés, disséminés dans le parenchyme.

\section{Discussion.}

L'examen histologique du foie, de la rate, du rein et du poumon de ces rats ne met pas en évidence de différence significative dans la répartition des altérations notées entre les animaux recevant l'huile d'arachide et ceux recevant l'huile de colza Primor.

En ce qui concerne les reins, les lésions de néphrite interstitielle chronique sont assez sévères pour des rats de cet âge (12 mois à l'autopsie), mais néanmoins banales chez le rat d'élevage. Elles correspondent aux altérations que nous observons chez des rats plus âgés (18 mois) de la souche OFA d'origine Sprague-Dawley élevés aux Oncins.

La rate de ces animaux apparaît congestive (saignée incomplète ?) et d'assez nombreux foyers d'hématopoïèse extramédullaire sont observés au niveau des cordons spléniques. D'après la litférature, cette hématopoï̀se splénique n'est pas rare chez le rat et pourrait être le résultat d'une affection intercurrente. Nous observons parfois des images semblables chez les rats de la souche OFA.

Enfin, aucune lésion majeure n'a été notée au niveau du poumon ou du foie. Les altérations pulmonaires sont discrètes et le foie a une structure histologique dans les limites normales pour des rats de 12 mois.

Effets physiologiques comparés des huiles de colza ef d'arachide. ATP-INRA, 1974-1976. 